\title{
Updated constraints on $f(T)$ models using direct and indirect measurements of the Hubble parameter
}

\author{
Spyros Basilakos ${ }^{a}$ Savvas Nesseris ${ }^{b}$ Fotios K. Anagnostopoulos ${ }^{a}$ Emmanuel N. \\ Saridakis ${ }^{c, d, e}$ \\ ${ }^{a}$ Academy of Athens, Research Center for Astronomy and Applied Mathematics, Soranou Efesiou 4, 11527, \\ Athens, Greece \\ ${ }^{b}$ Instituto de Física Teórica UAM-CSIC, Universidad Autonóma de Madrid, Cantoblanco, 28049 Madrid, \\ Spain \\ ${ }^{c}$ Department of Physics, National Technical University of Athens, Zografou Campus GR 157 73, Athens, \\ Greece \\ ${ }^{d}$ Chongqing University of Posts \& Telecommunications, Chongqing, 400065, China \\ ${ }^{e}$ CASPER, Physics Department, Baylor University, Waco, TX 76798-7310, USA \\ E-mail: svasileacademyofathens.gr, savvas.nesseris@csic.es, \\ fotis-anagnostopoulos@hotmail.com, \\ Emmanuel_Saridakisebaylor.edu
}

ABSTRACT: We extract observational constraints on $f(T)$ gravity, using the recently proposed statistical method which is not affected by the value of $H_{0}$ and thus it bypasses the problem of the disagreement in its exact numerical value between Planck and direct measurements. We use direct measurements of the Hubble parameter with the corresponding covariance matrix, and for completeness we perform a joint analysis using the latest data from Supernovae type Ia based on JLA sample, quasi-stellar objects, and Cosmic Microwave Background shift parameter from Planck. We analyze a large family of $f(T)$ models, and we compare the fitting results with $\Lambda$ CDM cosmology using the AIC statistical test. Utilizing only the Hubble parameter data we find that in the case of the power-law $f(T)$ model a small but non-zero deviation from $\Lambda \mathrm{CDM}$ cosmology is slightly favored at $1-\sigma$, nevertheless the corresponding AIC value shows a statistical equivalence with it. Finally, the join analysis reveals that all $f(T)$ models are very efficient and in very good agreement with observations.

KEYWORDS: $f(T)$ gravity, observational constraints, dark energy, Hubble parameter, quasi-stellar objects 


\section{Contents}

1 Introduction 1

$2 f(T)$ gravity and cosmology 2

3 Specific $f(T)$ models $\quad 4$

$4 \quad f(T)$ models against cosmological data $\quad 7$

$\begin{array}{lll}4.1 & H(z) \text { probes } & 7\end{array}$

4.2 Joint analysis with other probes 8

$\begin{array}{ll}\text { 4.2.1 Fitting } f(T) \text { models with } H(z) \text { data } & 10\end{array}$

$\begin{array}{ll}\text { 4.2.2 Fitting } f(T) \text { models with } H(z) \text {, standard candles and rulers } & 12\end{array}$

5 Constraining $f(T)$ models with future $H(z)$ data $\quad \mathbf{1 3}$

6 Conclusions 15

\section{Introduction}

The early and late time phases of accelerated expansion are amongst the most interesting findings of modern cosmology. In general, one may follow two main directions in order to offer an explanation. The first way is to keep general relativity as the theory of gravitational interactions and introduce new components such as the dark energy sector [1,2] and/or the inflation field(s) [3-5]. The second direction is to construct a modified theory of gravity, which possess general relativity as a particular limit, but with additional degrees of freedom that can drive acceleration [1, 6-9].

Most works in modified theories of gravity are based on the usual curvature-based formulation, and modify in various ways the Einstein-Hilbert action, as for instance in $f(R)$ gravity $[10,11]$. However, one can equally well construct gravitational modifications starting from the torsional formulation of gravity, and in particular from the Teleparallel Equivalent of General Relativity (TEGR) [12-16]. Since in this framework the gravitational Lagrangian is the torsion scalar $T$, the simplest torsional modified gravity would be to extend $T$ to $f(T)$, obtaining $f(T)$ gravity [17, 18] (see [19] for a review). Although TEGR is completely equivalent with general relativity at the level of equations, $f(T)$ gravity is different from $f(R)$ gravity, namely it is a novel class of gravitational modification, and that is why it has attracted the interest of the literature. The significant theoretical advantage of $f(T)$ gravity is that its field equations are always second-order, in contrast with $f(R)$ and other curvature-based modified theories of gravity. Concerning the cosmological implications, $f(T)$ gravity proves to lead to interesting phenomenology at both early [20, 21], as well as at late times [22-53].

Similarly to many models of modified gravity one can use observational data in order to constrain the large variety of $f(T)$ models that can be theoretically constructed. Hence, in the literature 
one can have works that use Solar System data [55], or data from Supernovae type Ia data (SNIa), Cosmic Microwave Background (CMB) shift parameters, Baryonic Acoustic Oscillations (BAO) growth rate and Hubble data observations [56-65] in order to extract constraints on the involved model parameters. At the cosmological level the above observations probe the integral of the Hubble parameter, and hence in the various analyses one must insert the current value of the Hubble function $H_{0}$. Unfortunately, the exact value of $H_{0}$ is a matter of debate due to the known disagreement between Planck [66] and local supernovae type Ia (hereafter SNIa; Riess et al. [67]) measurements.

In order to alleviate the aforementioned issue of the $H_{0}$ value, in [68] the authors presented a new statistical method which is not affected by the value of $H_{0}$. In particular, using the direct measurements of the Hubble expansion the so called $H(z)$ data [69], they marginalized analytically the Hubble constant in the likelihood function using Bayesian statistics. The latter approach has the property of circumvent the $H_{0}$ value problem and reducing the parameter space without adding numerical complexity. In the present work we desire to apply this $H_{0}$-independent method in order to impose constraints on various $f(T)$ models. Additionally, for completeness we perform a joint analysis using data from standard candles such as SNIa, and quasi-stellar objects (QSOs), and also CMB shift parameters from Planck [70]. We would like to stress that this is the first time that the $H_{0}$-independent method of the $H(z)$ data, the covariance matrix of the $H(z)$ measurements $[71,72]$, the JLA and the QSOs data are combined with the Planck CMB shift parameter toward constraining the $f(T)$ models.

The plan of the work is as follows: In section 2 we review $f(T)$ gravity and cosmology, and in section 3 we provide the basic specific $f(T)$ models that have appeared in the literature. In section 4 we present the method and the observational data sets that we use and we perform a detailed observational analysis providing the corresponding best fit values and contour plots for the various $f(T)$ models. In section $\mathrm{V}$, based on Monte Carlo simulations, we quantify the ability of future direct measurements of the Hubble parameter to place strong constraints on the $f(T)$ models. Finally, we discuss our conclusions in section VI.

\section{$2 f(T)$ gravity and cosmology}

In this section we briefly review $f(T)$ gravity and we apply it in a cosmological framework. In torsional formalism it proves convenient to use as dynamical variables the vierbeins fields $\mathbf{e}_{A}\left(x^{\mu}\right)$, which form an orthonormal basis for the tangent space at each point of the manifold $x^{\mu}$ (namely $\mathbf{e}_{A} \cdot \mathbf{e}_{B}=\eta_{A B}$, with $\eta_{A B}=\operatorname{diag}(1,-1,-1,-1)$. In a coordinate basis they are expressed as $\mathbf{e}_{A}=e_{A}^{\mu} \partial_{\mu}$ and therefore the metric is acquired as

$$
g_{\mu v}(x)=\eta_{A B} e_{\mu}^{A}(x) e_{\nu}^{B}(x)
$$

where Greek indices are used for the coordinate space-time while Latin indices for the tangent one.

One can now introduce the curvature-less Weitzenböck connection $\underset{\Gamma_{\nu \mu}^{\lambda}}{\mathbf{w}^{\lambda}} \equiv e_{A}^{\lambda} \partial_{\mu} e_{v}^{A}$ [73], in terms of which he can define the torsion tensor as

$$
T_{\mu \nu}^{\lambda}=\stackrel{\mathbf{w}}{\Gamma}_{\nu \mu}^{\lambda}-\stackrel{\mathbf{w}}{\Gamma}_{\mu \nu}^{\lambda}=e_{A}^{\lambda}\left(\partial_{\mu} e_{\nu}^{A}-\partial_{\nu} e_{\mu}^{A}\right)
$$


which contains all the information of the gravitational field. Contracting the torsion tensor we obtain the torsion scalar as

$$
T \equiv \frac{1}{4} T^{\rho \mu \nu} T_{\rho \mu \nu}+\frac{1}{2} T^{\rho \mu \nu} T_{\nu \mu \rho}-T_{\rho \mu}^{\rho} T_{\nu}^{v \mu},
$$

which is then used as the Lagrangian of teleparallel gravity (similarly to the use of the Ricci scalar as the Lagrangian of general relativity). Through variation of the teleparallel action in terms of the vierbeins, one obtains exactly the same equations with general relativity, and that is why the theory at hand was named teleparallel equivalent of general relativity (TEGR).

Inspired by the $f(R)$ extensions of general relativity, one can generalize $T$ to a function $T+$ $f(T)$. The resulting $f(T)$ theory of gravity is characterized by the action [19]

$$
S=\frac{1}{16 \pi G} \int d^{4} x e[T+f(T)],
$$

where $e=\operatorname{det}\left(e_{\mu}^{A}\right)=\sqrt{-g}$ and $G$ is the gravitational constant (the light speed is set to 1 for simplicity).

In order to study the cosmological applications of $f(T)$ gravity first we must add the matter and radiation sectors, and therefore the total action takes the form

$$
I=\frac{1}{16 \pi G} \int d^{4} x e\left[T+f(T)+L_{m}+L_{r}\right]
$$

Varying the above action with respect to the vierbeins we extract the field equations as

$$
e^{-1} \partial_{\mu}\left(e e_{A}^{\rho} S_{\rho}^{\mu \nu}\right)\left[1+f_{T}\right]+e_{A}^{\rho} S_{\rho}{ }^{\mu \nu} \partial_{\mu}(T) f_{T T}-\left[1+f_{T}\right] e_{A}^{\lambda} T^{\rho}{ }_{\mu \lambda} S_{\rho}{ }^{\nu \mu}+\frac{1}{4} e_{A}^{\nu}[T+f(T)]=4 \pi G e_{A}^{\rho^{\mathbf{e m}} T_{\rho}^{\nu},}
$$

where $f_{T}=\partial f / \partial T, f_{T T}=\partial^{2} f / \partial T^{2}$, and with $\stackrel{\text { em }}{T_{\rho}}{ }^{v}$ denoting the matter energy-momentum tensor. In the above equations we have introduced the "super-potential" $S_{\rho}{ }^{\mu \nu} \equiv \frac{1}{2}\left(K_{\rho}^{\mu \nu}+\delta_{\rho}^{\mu} T_{\alpha}^{\alpha \nu}-\delta_{\rho}^{\nu} T_{\alpha}^{\alpha \mu}\right)$, with $K_{\rho}^{\mu \nu} \equiv-\frac{1}{2}\left(T_{\rho}^{\mu \nu}-T_{\rho}^{\nu \mu}-T_{\rho}{ }^{\mu \nu}\right)$ the con-torsion tensor.

As a second step we impose the homogeneous and isotropic geometry $e_{\mu}^{A}=\operatorname{diag}(1, a, a, a)$, which corresponds to the spatially flat Friedmann-Robertson-Walker (FRW) metric

$$
d s^{2}=d t^{2}-a^{2}(t) \delta_{i j} d x^{i} d x^{j}
$$

where $a(t)$ is the scale factor. Inserting this vierbein choice into the field equations (2.6) we extract the Friedmann equations, namely

$$
\begin{aligned}
& H^{2}=\frac{8 \pi G}{3}\left(\rho_{m}+\rho_{r}\right)-\frac{f}{6}+\frac{T f_{T}}{3} \\
& \dot{H}=-\frac{4 \pi G\left(\rho_{m}+P_{m}+\rho_{r}+P_{r}\right)}{1+f_{T}+2 T f_{T T}},
\end{aligned}
$$

with $H \equiv \dot{a} / a$ the Hubble function, and where dots denote derivatives with respect to $t$ (note that we have used the fact that $T=-6 H^{2}$, which straightforwardly arises from (2.3) in FRW geometry). Moreover, in the above equations $\rho_{m}, \rho_{r}$ and $P_{m}, P_{r}$ are respectively the energy densities and pressures of the matter and radiation sectors, considered to correspond to perfect fluids. 
From the form of the Friedmann equations (2.8) and (2.9) it is implied that we can define the energy density and pressure of the effective dark energy sector as

$$
\begin{aligned}
& \rho_{D E} \equiv \frac{3}{8 \pi G}\left[-\frac{f}{6}+\frac{T f_{T}}{3}\right], \\
& P_{D E} \equiv \frac{1}{16 \pi G}\left[\frac{f-f_{T} T+2 T^{2} f_{T T}}{1+f_{T}+2 T f_{T T}}\right],
\end{aligned}
$$

and furthermore we can write its effective equation-of-state parameter as

$$
w \equiv \frac{P_{D E}}{\rho_{D E}}=-\frac{f / T-f_{T}+2 T f_{T T}}{\left[1+f_{T}+2 T f_{T T}\right]\left[f / T-2 f_{T}\right]} .
$$

Lastly, the equations close by considering the conservation equations of the matter and radiation sectors, namely

$$
\begin{gathered}
\dot{\rho}_{m}+3 H\left(\rho_{m}+P_{m}\right)=0 \\
\dot{\rho}_{r}+3 H\left(\rho_{r}+P_{r}\right)=0 .
\end{gathered}
$$

\section{Specific $f(T)$ models}

In the previous section we reviewed the basic equations of $f(T)$ gravity and cosmology. In this section we present some specific viable $f(T)$ models, and we provide the formalism to quantify their deviation from $\Lambda C D M$ cosmology in a unified way.

In order to elaborate the modified Friedmann equations we introduce

$$
E^{2}(z) \equiv \frac{H^{2}(z)}{H_{0}^{2}}=\frac{T(z)}{T_{0}}
$$

with $T_{0} \equiv-6 H_{0}^{2}$. We mention that it proves more convenient to use as the independent variable the redshift $z=\frac{a_{0}}{a}-1$, with $a_{0}$ the current scale factor set to one for simplicity (in the following the subscript " 0 " marks the current value of a quantity). Furthermore, assuming the matter to be dust, i.e. $w_{m} \equiv P_{m} / \rho_{m}=0$, from (2.13) we deduce that $\rho_{m}=\rho_{m 0}(1+z)^{3}$, and similarly imposing for the radiation $w_{r} \equiv P_{r} / \rho_{r}=1 / 3$ from (2.14) we acquire $\rho_{r}=\rho_{r 0}(1+z)^{4}$. Thus, the Friedmann equation (2.8) can be re-written as

$$
E^{2}(z, \mathbf{r})=\Omega_{m 0}(1+z)^{3}+\Omega_{r 0}(1+z)^{4}+\Omega_{F 0} y(z, \mathbf{r})
$$

with

$$
y(z, \mathbf{r})=\frac{1}{T_{0} \Omega_{F 0}}\left[f-2 T f_{T}\right] .
$$

In the above equations we have defined

$$
\Omega_{F 0}=1-\Omega_{m 0}-\Omega_{r 0},
$$

with $\Omega_{i 0}=\frac{8 \pi G \rho_{i 0}}{3 H_{0}^{2}}$ the value of the corresponding density parameter at present. In summary, the effect of $f(T)$ gravitational modification is quantified by the function $y(z, \mathbf{r})$, that is normalized to 
unity at present time, and which depends on $\Omega_{m 0}, \Omega_{r 0}$, and on the various parameters $r_{1}, r_{2}, \ldots$ that are involved in the specific $f(T)$ choice (assembled to form the vector $\mathbf{r}$ ). Note that in the limit of $\Lambda$ CDM cosmology, namely when $f(T)=$ const., the function $y(z, \mathbf{r})$ becomes a constant.

Let us now present all the specific $f(T)$ models that have been studied in the literature, that include two parameters one of which is independent. For each one of these models we calculate the function $y(z, \mathbf{r})$ through (3.3), and we quantify the deviation of $y(z, \mathbf{r})$ from its $\Lambda \mathrm{CDM}$ constant value using a distortion parameter $b$. The five $f(T)$ models are the following [59]:

1. The power-law model [17] (hereafter $f_{1} \mathrm{CDM}$ model), with

$$
f(T)=\alpha(-T)^{b},
$$

with $\alpha$ and $b$ the two parameters. Inserting this $f(T)$ form into (2.8) at current time we obtain

$$
\alpha=\left(6 H_{0}^{2}\right)^{1-b} \frac{\Omega_{F 0}}{2 b-1},
$$

while (3.3) gives

$$
y(z, b)=E^{2 b}(z, b) .
$$

Hence, when $b$ becomes zero the $f_{1} \mathrm{CDM}$ model reduces to $\Lambda \mathrm{CDM}$ cosmology, namely $T+$ $f(T)=T-2 \Lambda$, with $\Lambda=3 \Omega_{F 0} H_{0}^{2}$ and $\left.\Omega_{F 0}=\Omega_{\Lambda 0}\right)$.

2. The square-root exponential model (hereafter $f_{2} \mathrm{CDM}$ ) [18]

$$
f(T)=\alpha T_{0}\left(1-e^{-p \sqrt{T / T_{0}}}\right),
$$

with $\alpha$ and $p$ the two parameters. In this case (2.8) at present leads to

$$
\alpha=\frac{\Omega_{F 0}}{1-(1+p) e^{-p}}
$$

while (3.3) gives

$$
y(z, p)=\frac{1-(1+p E) e^{-p E}}{1-(1+p) e^{-p}} .
$$

Since $f_{2} \mathrm{CDM}$ reduces to $\Lambda \mathrm{CDM}$ cosmology for $p \rightarrow+\infty$, we can replace $p$ through $p=1 / b$ obtaining

$$
y(z, b)=\frac{1-\left(1+\frac{E}{b}\right) e^{-E / b}}{1-\left(1+\frac{1}{b}\right) e^{-1 / b}},
$$

which tends to constant (unity) for $b \rightarrow 0^{+}$.

3. The exponential model (hereafter $f_{3} \mathrm{CDM}$ ) [59]:

$$
f(T)=\alpha T_{0}\left(1-e^{-p T / T_{0}}\right),
$$

where $\alpha$ and $p$ are the two model parameters. In this case

$$
\alpha=\frac{\Omega_{F 0}}{1-(1+2 p) e^{-p}},
$$


while

$$
y(z, p)=\frac{1-\left(1+2 p E^{2}\right) e^{-p E^{2}}}{1-(1+2 p) e^{-p}} .
$$

We can re-write the above model using $p=1 / b$, resulting to

$$
y(z, b)=\frac{1-\left(1+\frac{2 E^{2}}{b}\right) e^{-E^{2} / b}}{1-\left(1+\frac{2}{b}\right) e^{-1 / b}},
$$

from which we can see that for $p \rightarrow+\infty$, or equivalently for $b \rightarrow 0^{+}$, the $f_{3} \mathrm{CDM}$ model reduces to $\Lambda \mathrm{CDM}$ cosmology.

4. The Bamba et al. logarithmic model (hereafter $f_{4} \mathrm{CDM}$ ) [74]

$$
f(T)=\alpha T_{0} \sqrt{\frac{T}{q T_{0}}} \ln \left(\frac{q T_{0}}{T}\right)
$$

with $\alpha$ and $q$ the two parameters. Equation (2.8) at present time gives

$$
\alpha=\frac{\Omega_{F 0} \sqrt{q}}{2},
$$

while (3.3) yields

$$
y(z)=E(z),
$$

Since the distortion function does not depend on the model parameters, we can re-write (3.2) as

$$
E(z)=\frac{1}{2} \sqrt{\Omega_{F 0}^{2}+4\left[\Omega_{m 0}(1+z)^{3}+\Omega_{r 0}(1+z)^{4}\right]}+\frac{\Omega_{F 0}}{2} .
$$

We mention that this model cannot reduce to $\Lambda \mathrm{CDM}$ cosmology for any value of its parameters.

5. The hyperbolic-tangent model (hereafter $f_{5} \mathrm{CDM}$ ) [75]:

$$
f(T)=\alpha(-T)^{n} \tanh \left(\frac{T_{0}}{T}\right)
$$

with $\alpha$ and $n$ the two parameters. In this case we acquire

$$
\alpha=-\frac{\Omega_{F 0}\left(6 H_{0}\right)^{1-n}}{\left[2 \operatorname{sech}^{2}(1)+(1-2 n) \tanh (1)\right]},
$$

and

$$
y(z, n)=E^{2(n-1)} \frac{2 \operatorname{sech}^{2}\left(\frac{1}{E^{2}}\right)+(1-2 n) E^{2} \tanh \left(\frac{1}{E^{2}}\right)}{2 \operatorname{sech}^{2}(1)+(1-2 n) \tanh (1)}
$$

Similarly to the previous model, the $f_{5} \mathrm{CDM}$ model cannot reduce to $\Lambda \mathrm{CDM}$ cosmology for any value of its parameters.

In summary, the above five $f(T)$ models are the ones with up to two parameters, out of which one is independent, that have been studied in the literature [59]. One could definitely consider also their combinations, however the appearance of many free parameters is not a desirable feature. Thus, in the following we investigate them separately. 


\section{$4 \quad f(T)$ models against cosmological data}

In this section we present the observational data and the statistical methods that we use in order to put constraints on the $f(T)$ models. In particular, we use direct measurements of the Hubble parameter, namely $H(z)$ data with the corresponding covariance matrix, the standard candles (SNIa and quasi-stellar objects: QSOs) and finally the CMB shift parameter data. Notice that in the case of the CMB shift parameter data, we need to include the contribution of the radiation term $\Omega_{r 0}$ in the normalized Hubble function. Here we utilize the following formula $\Omega_{r 0}=\Omega_{m 0} a_{\mathrm{e} q}$ [70], with $a_{\mathrm{e} q}=\frac{1}{1+2.5 \times 10^{4} h^{2}\left(T_{\mathrm{C} M B} / 2.7 K\right)^{-4}}$, where we have set $T_{\mathrm{C} M B}=2.7255 \mathrm{~K}$ and $h=0.68$.

\section{1 $H(z)$ probes}

Let us start with the $H(z)$ Hubble data set as compiled by Farooq et al., [69]. This sample contains $N=38$ entries in the following redshift interval $0.07 \leq z \leq 2.36$. The novelty here is that we use for the first time (to our knowledge) the covariance matrix of three BAO $H(z)$ measurements $[71,72]$ in constraining the $f(T)$ models.

Following standard lines, the nominal chi-square function is written as

$$
\chi_{H}^{2}\left(\phi^{\mu}\right)=\mathbf{V C}_{\mathrm{cov}}^{-1} \mathbf{V}^{T},
$$

where $\phi^{\mu}$ is the statistical vector that contains the free parameters, $\mathbf{C}_{\mathrm{cov}}^{-1}$ is the inverse of the covariance matrix [71, 72] and

$$
\mathbf{V}=\left\{H_{D}\left(z_{1}\right)-H_{M}\left(z_{1}, \phi^{\mu}\right), \ldots, H_{D}\left(z_{N}\right)-H_{M}\left(z_{N}, \phi^{\mu}\right)\right\} .
$$

Also, $z_{i}$ are the observed redshifts, while the letters $M$ and $D$ denote the data and models respectively. In this context, the theoretical Hubble parameter is parametrized as follows:

$$
H_{M}\left(z, \phi^{\mu}\right)=H_{0} E\left(z, \phi^{\mu+1}\right)
$$

and thus

$$
\mathbf{V}=\left\{H_{D}\left(z_{1}\right)-H_{0} E\left(z_{1}, \phi^{\mu+1}\right), . ., H_{D}\left(z_{N}\right)-H_{0} E\left(z_{N}, \phi^{\mu+1}\right)\right\},
$$

where $H_{0}$ is the Hubble constant, $E(z)$ is the dimensionless Hubble function and the vector $\phi^{\mu+1}$ contains the other free parameters of the $f(T)$ models, namely $\left(\Omega_{m 0}, b\right)$ etc.

Clearly, using the usual $\chi^{2}$ estimator of Eq.(4.1) in constraining the $f(T)$ gravity models we have to either impose the exact value of $H_{0}$ or treating it as a free parameter. The first option is rather inconvenient due to the well known Hubble constant problem, namely the observed Hubble constant $\left(H_{0}=73.24 \pm 1.74 \mathrm{Km} / \mathrm{s} / \mathrm{Mpc}\right)$ found by the SNIa team (Riess et al. [67]) is in $\sim 3-3.5 \sigma$ tension with that of Planck (see $H_{0}=67.8 \pm 0.9 \mathrm{Km} / \mathrm{s} / \mathrm{Mpc}$; [66]). On the other hand, if we treat $H_{0}$ as a free parameter then we increase the parameter space. Recently, [68] proposed a novel statistical technique towards overcoming the above problems. Here we present the main points of this method. Specifically, inserting the vector (4.3) into Eq. (4.1) we find after some simple calculations

$$
\chi^{2}\left(\phi^{\mu}\right)=A H_{0}^{2}-2 B H_{0}+\Gamma
$$


where

$$
\begin{aligned}
A & =\mathbf{E C}_{\mathrm{cov}}^{-1} \mathbf{E}^{T}, \\
B & =\frac{1}{2}\left(\mathbf{E C}_{\mathrm{cov}}^{-1} \mathbf{H}_{\mathrm{D}}^{T}+\mathbf{H}_{D} \mathbf{C}_{\mathrm{cov}}^{-1} \mathbf{E}^{T}\right) \\
\Gamma & =\mathbf{H}_{D} \mathbf{C}_{\mathrm{c} o v}^{-1} \mathbf{H}_{D}^{T},
\end{aligned}
$$

with

$$
\mathbf{E}=\left\{E\left(z_{1}, \phi^{\mu+1}\right), \ldots, E\left(z_{N}, \phi^{\mu+1}\right)\right\}
$$

and

$$
\mathbf{H}_{D}=\left\{H_{D}\left(z_{1}\right), \ldots, H_{D}\left(z_{N}\right)\right\} .
$$

Therefore, the likelihood function of $\chi^{2}$, namely $\mathcal{L}=e^{-\chi^{2} / 2}$, is written as

$$
\mathcal{L}\left(D \mid \phi^{\mu}, M\right)=\exp \left[\frac{A\left(H_{0}-\frac{B}{A}\right)^{2}-\frac{B^{2}}{A}+\Gamma}{2}\right] .
$$

Marginalizing over $H_{0}$ in the context of Bayes's theorem we find

$$
p\left(\phi^{\mu} \mid D, M\right)=\frac{1}{p(D \mid M)} \int e^{-\frac{A\left(H_{0}-B / A\right)^{2}-B^{2} / A+\Gamma}{2}} d H_{0} .
$$

Moreover, using the new variable $y=H_{0}-B / A$, assuming that $H_{0} \in(0,+\infty)$ and introducing flat priors $p\left(\phi^{\mu} \mid M, H_{0}\right)=1$, we arrive at

$$
p\left(\phi^{\mu} \mid D, M\right)=\frac{1}{p(D \mid M)} e^{-\frac{1}{2}\left(\Gamma-\frac{B^{2}}{A}\right)} \sqrt{\frac{\pi}{2 A}}\left[1+\mathrm{erf}\left(\frac{B}{\sqrt{2 A}}\right)\right],
$$

where $\operatorname{er} f(x)=\frac{2}{\sqrt{\pi}} \int_{0}^{x} e^{-y^{2}} d y$ is the error function. To this end, it is trivial to show that the above likelihood function reduces to a new $\tilde{\chi}_{H}^{2}$ function which is written as

$$
\tilde{\chi}_{H}^{2}\left(\phi^{\mu+1}\right)=\Gamma-\frac{B^{2}}{A}+\ln A-2 \ln \left[1+\operatorname{erf}\left(\frac{B}{\sqrt{2 A}}\right)\right] .
$$

Notice that in the latter expression the constant $\ln (\pi / 2)$, is ignored since it does not contribute in the minimization procedure.

Evidently, the estimator $\tilde{\chi}_{H}^{2}$ alleviates the Hubble constant problem since it is not affected by $H_{0}$. We would like to stress that this is the first time that the current approach is implemented towards testing the performance of the $f(T)$ models against the $H(z)$ data.

\subsection{Joint analysis with other probes}

In order to place tight constraints on the model parameters we use a joint likelihood analysis, involving the standard candles (SNIa and QSOs, hereafter SC) and standard rulers (CMB shift parameter) together with the $H(z)$ data. Notice, that standard candles and rulers probe the integral of the Hubble parameter $H(z)$, implying that they give indirect information of the cosmic expansion, while the $H(z)$ data provide direct measurements of the expansion rate of the universe. Therefore, 


\begin{tabular}{|c|c|c|c|c|c|c|c|c|}
\hline Data & Model & $\Omega_{m 0}$ & $b$ & $\alpha$ & $\beta$ & $\chi_{\min }^{2}$ & $\mathrm{~A} I C$ & $|\triangle \mathrm{AIC}|$ \\
\hline \multirow{4}{*}{$H(z):$} & $f_{1} \mathrm{CDM}$ & $0.229 \pm 0.072$ & $0.584 \pm 0.377$ & - & - & 18.967 & 23.310 & 0.207 \\
\hline & $f_{2} \mathrm{CDM}$ & $0.284 \pm 0.029$ & $0.600 \pm 0.410$ & - & - & 19.363 & 23.706 & 0.603 \\
\hline & $f_{3} \mathrm{CDM}$ & $0.297 \pm 0.077$ & $0.266 \pm 0.169$ & - & - & 20.741 & 25.084 & 1.981 \\
\hline & $\Lambda \mathrm{CDM}$ & $0.265 \pm 0.023$ & - & - & - & 20.992 & 23.103 & 0.000 \\
\hline \multirow{4}{*}{$H(z) / \mathrm{SC}:$} & $f_{1} \mathrm{CDM}$ & $0.249 \pm 0.029$ & $0.258 \pm 0.253$ & $0.141 \pm 0.058$ & $3.102 \pm 0.635$ & 738.836 & 746.886 & 0.552 \\
\hline & $f_{2} \mathrm{CDM}$ & $0.263 \pm 0.021$ & $0.301 \pm 0.148$ & $0.141 \pm 0.059$ & $3.102 \pm 0.650$ & 739.022 & 747.072 & 0.738 \\
\hline & $f_{3} \mathrm{CDM}$ & $0.267 \pm 0.022$ & $0.194 \pm 0.069$ & $0.141 \pm 0.069$ & $3.104 \pm 0.769$ & 739.441 & 747.491 & 1.157 \\
\hline & $\Lambda \mathrm{CDM}$ & $0.269 \pm 0.021$ & - & $0.142 \pm 0.041$ & $3.112 \pm 0.474$ & 740. 304 & 746.334 & 0.000 \\
\hline \multirow{4}{*}{$H(z) / \mathrm{SC} / \mathrm{CMB}_{\text {shift }}:$} & $f_{1} \mathrm{CDM}$ & $0.307 \pm 0.003$ & $-0.018 \pm 0.029$ & $0.141 \pm 0.033$ & $3.101 \pm 0.414$ & 743.964 & 752.014 & 1.546 \\
\hline & $f_{2} \mathrm{CDM}$ & $0.305 \pm 0.001$ & $0.047 \pm 0.082$ & $0.141 \pm 0.033$ & $3.101 \pm 0.412$ & 744.438 & 752.488 & 2.020 \\
\hline & $f_{3} \mathrm{CDM}$ & $0.305 \pm 0.001$ & $0.050 \pm 0.061$ & $0.142 \pm 0.032$ & $3.100 \pm 0.203$ & 744.446 & 752.496 & 2.028 \\
\hline & $\Lambda \mathrm{CDM}$ & $0.305 \pm 0.001$ & - & $0.141 \pm 0.033$ & $3.101 \pm 0.412$ & 744.438 & 750.468 & 0.000 \\
\hline
\end{tabular}

Table 1. Cosmological constraints. The first column indicates the data set (s) used, the second column includes the cosmological models used in this study the third and fourth columns provide the $\Omega_{m 0}$ and $b$ best fit values. The next two columns show the values of the JLA-data intrinsic free parameters, namely $\alpha$ and $\beta$. The last three columns present the goodness-of-fit statistics $\left(\chi_{\min }^{2}, \mathrm{AIC}\right.$ and $\left.\triangle \mathrm{AIC}=\mathrm{A} I C_{\mathrm{model}}-\mathrm{A} I C_{\mathrm{min}} \mid\right)$. The abbreviation SC denotes standard candles, namely SNIa and QSOs.

the combination of the latter observational probes appears as an ideal tool in constraining the $f(T)$ gravity models.

Specifically, we utilize the JLA sample of 740 SN Ia of Betoule et al. [76] and the binned dataset of QSOs [77, 78] that contains 24 entries. It is interesting to mention that combining the SNIa data with those of QSOs we manage to trace the Hubble relation (distance modulus versus $z$ ) in the redshift range $0.07<z<6$.

Regarding the chi-square function of the standard candles $\chi_{\mathrm{SC}}^{2}$ we use the estimator of [79] which is not affected by the value of the Hubble constant (see also Ref.[80] and references therein), while for QSOs we refer the reader the recent work of [78]. Moreover, we utilize the position of the CMB shift of acoustic peaks provided by the Planck 2015 data points $\left(l_{a}, R, z_{*}\right)$. The chi-square $\chi_{\mathrm{C} M B}^{2}$, the CMB shift parameter data, the theoretical formulas of $\left(l_{a}, R, z_{\star}\right)$, the value of $\Omega_{r 0}$ and the inverse of the corresponding covariance matrix can be found in Ref. [70].

Since the total likelihood function $\mathcal{L}_{\text {tot }}$ is defined as the product of the individual likelihoods, namely

$$
\mathcal{L}_{\mathrm{t} o t}=\mathcal{L}_{H} \times \mathcal{L}_{\mathrm{S} C} \times \mathcal{L}_{\mathrm{CMB}}
$$

it is implied that

$$
\chi_{\mathrm{t} o t}^{2}=\tilde{\chi}_{H}^{2}+\chi_{\mathrm{SC}}^{2}+\chi_{\mathrm{C} M B}^{2} .
$$

In order to test the statistical significance of our constraints we implement the AIC [81] criterion. In particular, considering Gaussian errors the corresponding estimator is

$$
\mathrm{AIC}=-2 \ln \mathcal{L}_{\max }+2 k+\frac{2 k(k+1)}{N-k-1},
$$

where $N$ and $k$ denote the total number of data and the number of fitted parameters (see also [82]). Obviously, a smaller value of AIC means a better model-data fit. In this framework, if we want 


\begin{tabular}{|c|c|c|c|c|c|c|c|c|c|}
\hline Data & Model & $\overline{\Omega_{m 0}}$ & $\bar{b}$ & $\bar{\alpha}$ & $\bar{\beta}$ & $\bar{h}$ & $\chi_{\min }^{2}$ & $\overline{\mathrm{A} I C}$ & $|\Delta \mathrm{AIC}|$ \\
\hline \multirow{4}{*}{$H(z):$} & $f_{1} \mathrm{CDM}$ & $0.226 \pm 0.077$ & $0.603 \pm 0.374$ & - & - & $0.651 \pm 0.037$ & 19.768 & 26.474 & 0.205 \\
\hline & $f_{2} \mathrm{CDM}$ & $0.285 \pm 0.029$ & $0.622 \pm 0.426$ & - & - & $0.644 \pm 0.039$ & 20.145 & 26.851 & 0.582 \\
\hline & $f_{3} \mathrm{CDM}$ & $0.310 \pm 0.108$ & $0.292 \pm 0.199$ & - & - & $0.639 \pm 0.115$ & 21.529 & 28.235 & 1.966 \\
\hline & $\Lambda \mathrm{CDM}$ & $0.266 \pm 0.018$ & - & - & - & $0.696 \pm 0.014$ & 21.926 & 26.269 & 0.000 \\
\hline \multirow{4}{*}{$H(z) / \mathrm{SC}:$} & $f_{1} \mathrm{CDM}$ & $0.249 \pm 0.028$ & $0.260 \pm 0.234$ & $0.141 \pm 0.055$ & $3.103 \pm 0.614$ & $0.686 \pm 0.020$ & 739.740 & 749.815 & 0.534 \\
\hline & $f_{2} \mathrm{CDM}$ & $0.264 \pm 0.021$ & $0.302 \pm 0.134$ & $0.141 \pm 0.055$ & $3.103 \pm 0.621$ & $0.686 \pm 0.020$ & 739.926 & 750.001 & 0.720 \\
\hline & $f_{3} \mathrm{CDM}$ & $0.267 \pm 0.022$ & $0.194 \pm 0.066$ & $0.141 \pm 0.065$ & $3.104 \pm 0.735$ & $0.687 \pm 0.021$ & 740.351 & 750.426 & 1.145 \\
\hline & $\Lambda \mathrm{CDM}$ & $0.269 \pm 0.021$ & - & $0.142 \pm 0.041$ & $3.112 \pm 0.474$ & $0.694 \pm 0.016$ & 741.231 & 749.281 & 0.000 \\
\hline \multirow{4}{*}{$H(z) / \mathrm{SC} / \mathrm{CMB}_{\text {shift }}:$} & $f_{1} \mathrm{CDM}$ & $0.307 \pm 0.003$ & $-0.020 \pm 0.028$ & $0.142 \pm 0.007$ & $3.101 \pm 0.080$ & $0.671 \pm 0.007$ & 744.804 & 754.879 & 1.466 \\
\hline & $f_{2} \mathrm{CDM}$ & $0.305 \pm 0.001$ & $0.040 \pm 0.080$ & $0.141 \pm 0.006$ & $3.100 \pm 0.080$ & $0.671 \pm 0.007$ & 745.357 & 755.432 & 2.019 \\
\hline & $f_{3} \mathrm{CDM}$ & $0.305 \pm 0.001$ & $0.054 \pm 0.072$ & $0.142 \pm 0.007$ & $3.097 \pm 0.080$ & $0.671 \pm 0.007$ & 745.365 & 755.440 & 2.027 \\
\hline & $\Lambda \mathrm{CDM}$ & $0.305 \pm 0.001$ & - & $0.142 \pm 0.007$ & $3.100 \pm 0.080$ & $0.671 \pm 0.007$ & 745.363 & 753.413 & 0.000 \\
\hline
\end{tabular}

Table 2. Same as Table 1, however now the parameter $h \equiv \frac{H_{0}}{100 \mathrm{kms}^{-1} \mathrm{Mpc}^{-1}}$ is not marginalized over, but it is free to vary.

to test the performance of the different cosmological models in fitting the observational data then we need to introduce the model pair difference, namely $\triangle \mathrm{A} I C=\mathrm{A} I C_{\text {model }}-\mathrm{A} I C_{\min }$. Therefore, the inequalities $4<\triangle \mathrm{AIC}<7$ indicate a positive evidence against the model with higher value of $\mathrm{AIC} C_{\text {model }}[83,84]$, while the restriction $\triangle \mathrm{AIC} \geq 10$ suggests a strong such evidence. On the other hand, the condition $\triangle \mathrm{A} I C \leq 2$ implies an indication of consistency between the two comparison models.

In summary, an overall presentation of our constraints is listed in Table 1 for various cosmological probes. The Table contains the fitted model parameters, including the intrinsic values of JLA $(\alpha, \beta)$, and the goodness of fit statistics $\left(\chi_{\min }^{2}\right.$, AIC), for the specific $f(T)$ gravity models. For comparison we additionally provide the results of the usual $\Lambda \mathrm{CDM}$ cosmological model. Furthermore, in Table 2 we show the results after the parameter $H_{0}$ has been allowed to vary. Comparing the two Tables we see that our results and the two methods of analysis are perfectly consistent with each other. Furthermore, from Table 2 we observe that the $f(T)$ models support a smaller value of Hubble constant than the value obtained from Cepheids. In particular, for all $f(T)$ models we find $H_{0}=67.1 \pm 0.7 \mathrm{Km} / \mathrm{s} / \mathrm{Mpc}$ which is closer to the Planck value.

Below, we provide a qualitative discussion of our constraints, giving the reader the opportunity to appreciate the new results of our study. Notice that we have excluded models $f_{4} \mathrm{CDM}$ and $f_{5} \mathrm{CDM}$ from the rest of the analysis, since we have confirmed the results of Nesseris et al. [59], namely that the observational data disfavor the above two $f(T)$ models at high significance level.

\subsubsection{Fitting $f(T)$ models with $H(z)$ data}

In the light of the new chi-square estimator [see Eq.(4.7)] we utilize for the first time the covariance matrix of $H(z)$ data $[71,72]$ in order to constrain the $f(T)$ models. Let us now briefly present our results. For comparison we also provide the results of Nunes et al. [61].

Specifically, we find:

- For $f_{1}$ CDM model: $\tilde{\chi}_{H}^{2}=18.967$ (AIC $\left.=23.310\right), \Omega_{m 0}=0.229 \pm 0.072$ and $b=0.584 \pm 0.377$, while [61] found: $\left(\Omega_{m 0}, b\right)=\left(0.231_{-0.019}^{+0.016}, 0.033_{-0.035}^{+0.045}\right)$ for $H_{0}=72.85_{-1.8}^{+1.7} \mathrm{Km} / \mathrm{s} / \mathrm{Mpc}$. 

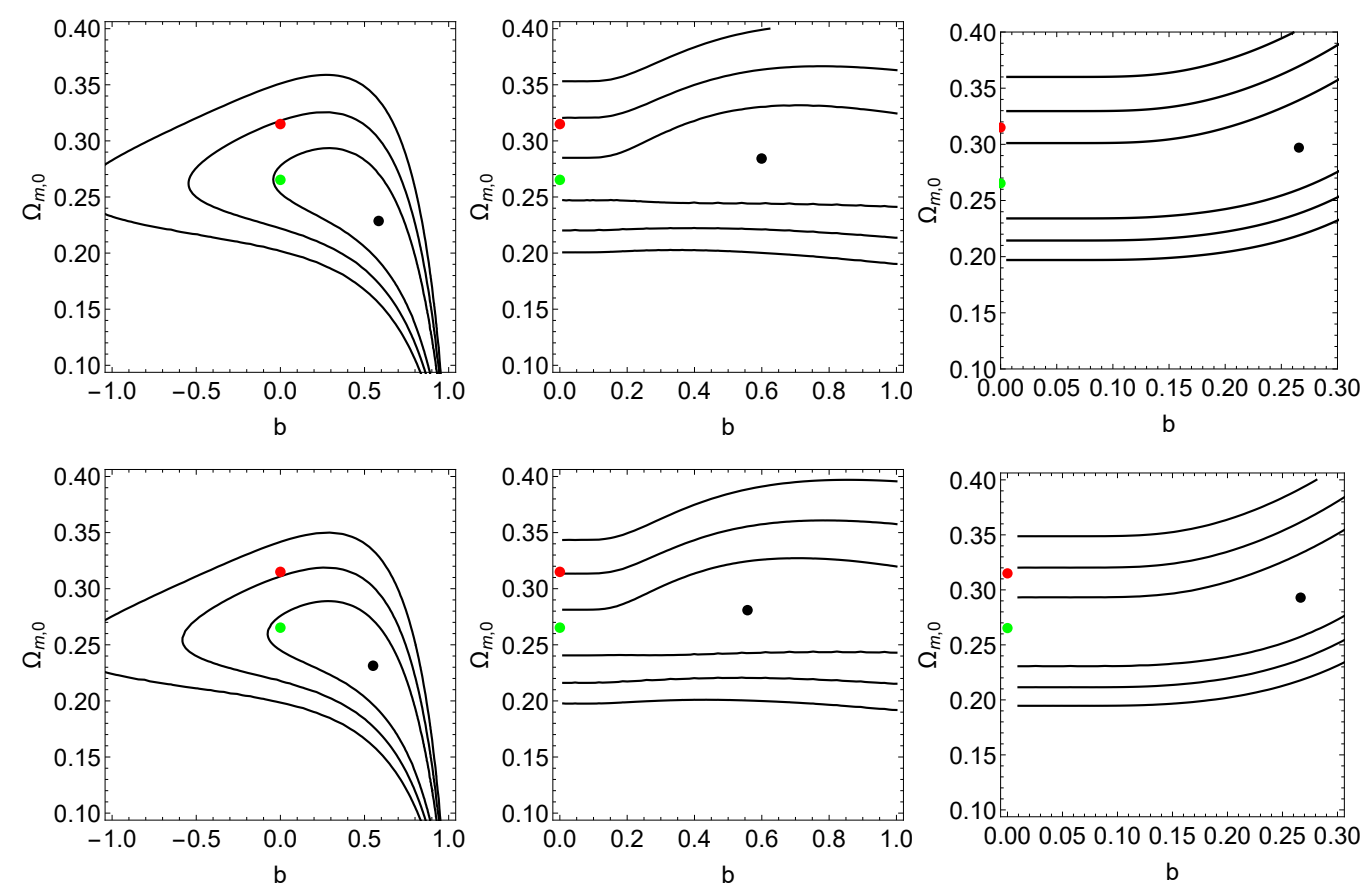

Figure 1. Likelihood contours in the $b-\Omega_{m 0}$ plane using the $H(z)$ data. The contours correspond to $1 \sigma, 2 \sigma$ and $3 \sigma$ confidence levels. From left to right, observational constraints for power-law $f_{1} \mathrm{CDM}(3.5), f_{2} \mathrm{CDM}$ model (3.8) and $f_{3} \mathrm{CDM}$ model (3.12). From bottom to top, constraints using $H(z)$ data without covariance and adding covariance. The black point represents the best fit solutions for the current $f(T)$ models (see Table I). Notice, that the red (or green) dot point corresponds to the flat $\Lambda$ CDM model using the Planck [66] [or $H(z)$ ] best fit solution.

- For $f_{2} \mathrm{CDM}$ model: $\tilde{\chi}_{H}^{2}=19.363$ (AIC=23.706), $\Omega_{m 0}=0.284 \pm 0.029$ and $b=0.600 \pm$ 0.410. Notice that [61] obtained: $\left(\Omega_{m 0}, b\right)=\left(0.243_{-0.015}^{+0.014}, 0.112_{-0.035}^{+0.045}\right)$ for $H_{0}=71.53 \pm 1.3$ $\mathrm{Km} / \mathrm{s} / \mathrm{Mpc}$.

- For $f_{3} \mathrm{CDM}$ model: $\tilde{\chi}_{H}^{2}=20.741$ (AIC $\left.=25.084\right), \Omega_{m 0}=0.297 \pm 0.077$ and $b=0.266 \pm 0.169$. To this end, [61] found: $\left(\Omega_{m 0}, b\right)=\left(0.242_{-0.015}^{+0.013}, 0.106_{-0.090}^{+0.052}\right)$ for $H_{0}=71.57 \pm 1.3 \mathrm{Km} / \mathrm{s} / \mathrm{Mpc}$.

- For $\Lambda$ CDM model: $\tilde{\chi}_{H}^{2}=20.992(\mathrm{AIC}=23.103)$ and $\Omega_{m 0}=0.265 \pm 0.023$.

In Fig. 1 we present the $1 \sigma, 2 \sigma$ and $3 \sigma$ contours in the $\left(b, \Omega_{m 0}\right)$ plane for the $f_{1-3} \mathrm{CDM}$ models. Taking into account the best- $\tilde{\chi}^{2}$ and the value of AIC we find that the best model is the $f_{1} \mathrm{CDM}$, while there is a mild tension between the $f_{3} \mathrm{CDM}$ models and the $H(z)$ data $\triangle \mathrm{AIC}=$ $\mathrm{AIC} C_{f_{1}}-\mathrm{AIC} C_{f_{3}}>2$. Moreover, the fact $\triangle \mathrm{AIC} \leq 2$ implies that the $f_{1} \mathrm{CDM}$ model is statistically equivalent with $\Lambda \mathrm{CDM}$ and $f_{2} \mathrm{CDM}$.

Lastly, we would like to stress that the aforementioned $\Omega_{m 0}$ constraints are in agreement within $1 \sigma$ errors with those of Nunes et al. [61] who considered the case where the covariance matrix of the $H(z)$ data is diagonal. However, our results regarding the $b$ parameters of $f_{1,2} \mathrm{CDM}$ models are somewhat larger $(\sim 1.2-1.5 \sigma)$ with those of [61], while in the case of $f_{3} \mathrm{CDM}$ model the constraints are similar (within $1 \sigma$ ) in both studies. We mention that in the work of Nunes et al. [61] the cosmic chronometer data, which are based on the relative ages of the passively evolving galaxies, were 
used. This sample contains the $30 H(z)$ measurements in the redshift range $0<z<2$. Moreover, Nunes et al. [61] used the standard estimator (4.1) and thus they had to treat the value of $H_{0}$ as a free parameter. In order to understand the effectiveness of the $H(z)$ covariance matrix and the new chi-square estimator in constraining the $b-\Omega_{m 0}$ solution space, we compare the $b-\Omega_{m 0}$ contours with and without covariance.

\subsubsection{Fitting $f(T)$ models with $H(z)$, standard candles and rulers}

Firstly, we combine the observed Hubble relation provided by SNIa and QSOs data and the direct measurements of the Hubble parameter. The results are as follows. Regarding the $f(T)$ models we obtain $\left(\Omega_{m 0}, b\right)=(0.249 \pm 0.029,0.258 \pm 0.253),\left(\Omega_{m 0}, b\right)=(0.263 \pm 0.021,0.301 \pm 0.148)$ and $\left(\Omega_{m 0}, b\right)=(0.267 \pm 0.022,0.194 \pm 0.069)$ for the $f_{1} \mathrm{CDM}, f_{2} \mathrm{CDM}$ and $f_{3} \mathrm{CDM}$ models, respectively, with $\chi_{\min }^{2}=738.836, \chi_{\min }^{2}=739.022$ and $\chi_{\min }^{2}=739.441$. The latter constraints are in agreement within $1 \sigma$ (within $1 \sigma$ uncertainties) with those of Nunes et al. [61] who found, combing cosmic chronometer, SNIa (Union 2.1) and BAO data, $\left(\Omega_{m 0}, b\right)=\left(0.234_{-0.014}^{+0.016}, 0.051_{-0.019}^{+0.025}\right)$ with $H_{0}=$ $72.75_{-1.8}^{+1.7} \mathrm{Km} / \mathrm{s} / \mathrm{Mpc}$ for $f_{1} \mathrm{CDM},\left(\Omega_{m 0}, b\right)=\left(0.278_{-0.02}^{+0.01}, 0.133_{-0.130}^{+0.043}\right)$ with $H_{0}=68.19_{-0.93}^{+1.90} \mathrm{Km} / \mathrm{s} / \mathrm{Mpc}$ for $f_{2} \mathrm{CDM}$ and $\left(\Omega_{m 0}, b\right)=\left(0.266_{-0.010}^{+0.009}, 0.09_{-0.08}^{+0.041}\right)$ with $H_{0}=69.8_{-0.84}^{+0.89} \mathrm{Km} / \mathrm{s} / \mathrm{Mpc}$ for $f_{3} \mathrm{CDM}$ models respectively.

Including the CMB shift parameters data in the likelihood analysis we find $\left(\Omega_{m 0}, b\right)=(0.307 \pm$ $0.003,-0.018 \pm 0.029),\left(\Omega_{m 0}, b\right)=(0.305 \pm 0.001,0.047 \pm 0.082)$ and $\left(\Omega_{m 0}, b\right)=(0.305 \pm 0.001,0.050 \pm$ 0.061 ) for the $f_{1} \mathrm{CDM}, f_{2} \mathrm{CDM}$ and $f_{3} \mathrm{CDM}$ models, respectively, with $\chi_{\min }^{2}$ of $\sim 744$. Evidently, using the joint cases we find that the best model is $\Lambda$ CDM. Based on AIC we observe that the $f_{1}, f_{2}, f_{3}$ models fit at a statistically acceptable level either the $H(z)$ or $H(z) / \mathrm{SNIa} / \mathrm{QSO}$ data.

In contrast, there is a weak evidence ( $\triangle \mathrm{AIC} \sim 2$ ) against $f_{2,3} \mathrm{CDM}$ from the $H(z) / \mathrm{SNIa} / \mathrm{QSOs} /$ $\mathrm{CMB}_{\text {shift }}$ data, while the fact $\triangle \mathrm{AIC}<2$ implies that the power-law $f_{1} \mathrm{CDM}$ model is statistically equivalent with that of $\Lambda \mathrm{CDM}$. Furthermore, we would like to point that our results are in agreement (within $1 \sigma$ errors) with those of Nesseris et al. [59] who used a combination of $\mathrm{SNIa} / \mathrm{BAO} / \mathrm{CMB}_{\text {shift }}$ data. Concerning the $f_{1} \mathrm{CDM}$ model the present results can be compared with those of Nunes (2018) [64] who used the CMB power spectrum. Lastly, our constraints for $f_{1} \mathrm{CDM}$ and $f_{2} \mathrm{CDM}$ models are in very good agreement with those of and $\mathrm{Xu}$ et al. [65] who

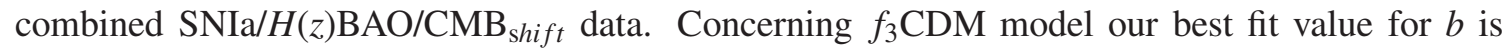
somewhat lower with that of [65]. Specifically, these authors found: (a) $H_{0}=69.4 \pm 0.8 \mathrm{Km} / \mathrm{s} / \mathrm{Mpc}$, $\Omega_{m 0}=0.298 \pm 0.007$ and $b=-0.10_{-0.07}^{+0.09}$ for $f_{1} \mathrm{CDM}$ model, (b) $H_{0}=69.6 \pm 0.9 \mathrm{Km} / \mathrm{s} / \mathrm{Mpc}$, $\Omega_{m 0}=0.296 \pm 0.007$ and $b=0.13_{-0.11}^{+0.09}$ for $f_{2} \mathrm{CDM}$ model, and (c) $H_{0}=69.5 \pm 0.8 \mathrm{Km} / \mathrm{s} / \mathrm{Mpc}$, $\Omega_{m 0}=0.297 \pm 0.007$ and $b=0.41 \pm 0.31$ in the case of $f_{3}$ CDM model.

Notice, in Figs. 2 and 3 we show the corresponding confidence contours. Comparing the first panel with the other two, we see that the Figure-of-Merit (FoM) ${ }^{1}$ of $f(T)$ models is increased by a factor of $\sim 2.5$ in the case of $H(z) / \mathrm{SNIa} / \mathrm{QSOs}$, while for $H(z) / \mathrm{SNIa} / \mathrm{QSOs} / \mathrm{CMB}_{\text {shift }}$ we find a two-fold increase of FoM.

Finally, in order to give the reader the opportunity to appreciate the new results of our study we conclude this section with a brief discussion regarding our new and novel statistical results.

\footnotetext{
${ }^{1}$ The FoM is defined as the inverse of the enclosed area of the $2 \sigma$ contour in the solution space of any two degenerate free parameters, is our case $b-\Omega_{m 0}$.
} 

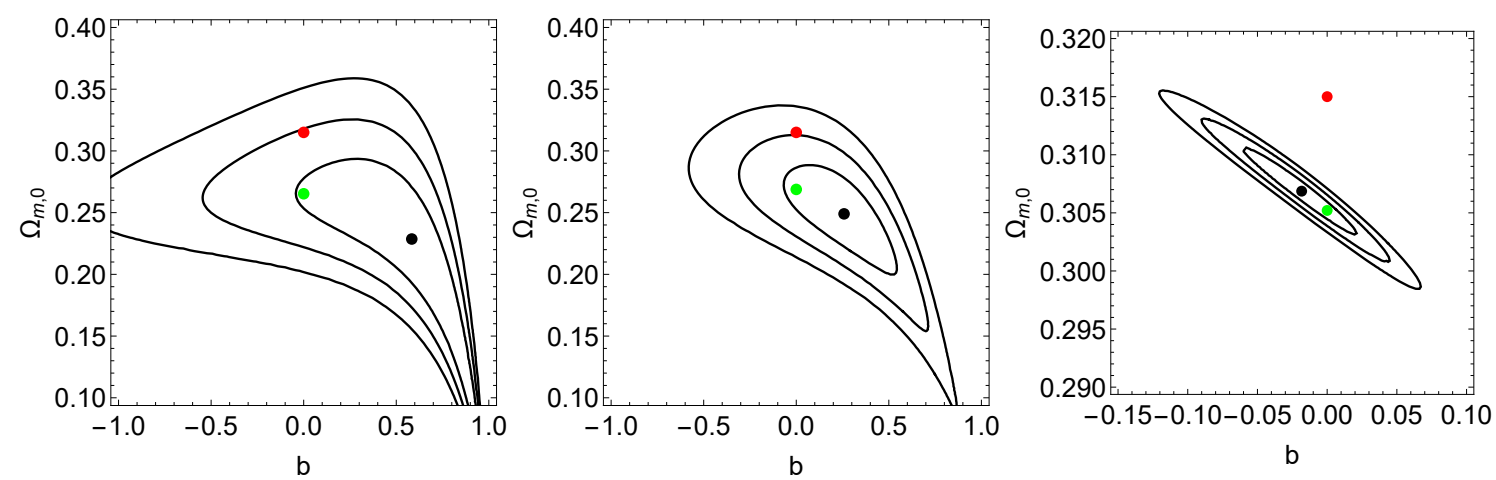

Figure 2. Likelihood contours in the case of the power-law $f_{1} \mathrm{CDM}$ model, using (left to right) the following data: (a) $H(z)$ data, (b) $H(z) / \mathrm{QSO} / \mathrm{SNIa}$ and (c) $H(z) / \mathrm{QSO} / \mathrm{SNIa} / \mathrm{CMB}_{\text {shift }}$. The red point corresponds to the Planck $\Lambda$ CDM model [66], while our best fit solutions are given by green $(\Lambda \mathrm{CDM})$ and black $\left(f_{1} \mathrm{CDM}\right)$ points respectively.

Although, our observational constraints are in qualitative agreement with previous studies on $f(T)$ gravity [56-60, 62-65], we would like to spell out clearly the reasons of which our analysis improves the observational constraints of $f(T)$ models with respect to previous studies. Firstly, we include for the first time the covariance matrix of $H(z)$ data in $f(T)$ models, and we utilize at the same time the recently proposed statistical method of [68], which is not affected by the Hubble constant problem. Secondly, we use the total amount of standard candles, namely we combine the SNIa data with those of QSOs and thus we trace the Hubble relation in the redshift range $0.07<z<6$.

\section{Constraining $f(T)$ models with future $H(z)$ data}

As we have already discussed the statistical analysis of section 4.B.1 indicates that direct measurements of the Hubble expansion favor the power law $f(T)$ model over the other models, including that of $\Lambda \mathrm{CDM}$. In this section we investigate the impact of using future Hubble parameter data, based on the next generation of surveys, to distinguish the power law $f(T)$ model from the expectations of $\Lambda \mathrm{CDM}$.

In particular, we are interested to check how better can we go in constraining the $f(T)$ models by increasing the number of the present $H(z)$ measurements from 38 to 100. A detailed discussion concerning this test can been found in [68], where the authors applied their algorithm on the scalarfield dark energy models. Specifically, we generate a large sets of Monte Carlo simulations towards quantifying the ability of future $H(z)$ data to place strong constraints on the $f(T)$ models. Notice that this is the first time in the literature that such an analysis is applied on the $f(T)$ gravity models. The algorithm is developed via a three-step process, a brief description of which is as follows.

- Firstly, we select the viable $f_{1} \mathrm{CDM}$ model with $\left(\Omega_{m 0}, b, H_{0}\right)=(0.214,0.653,68)$ as a reference model.

- Secondly, we peak a redshift $z_{\mathrm{r} a n} \in[0.07,2.36]$, by randomly sampling the redshift distribution of the current $H(z)$ sample, and we extract the measured Hubble parameter $H_{D}\left(z_{\text {ran }}\right)$ 

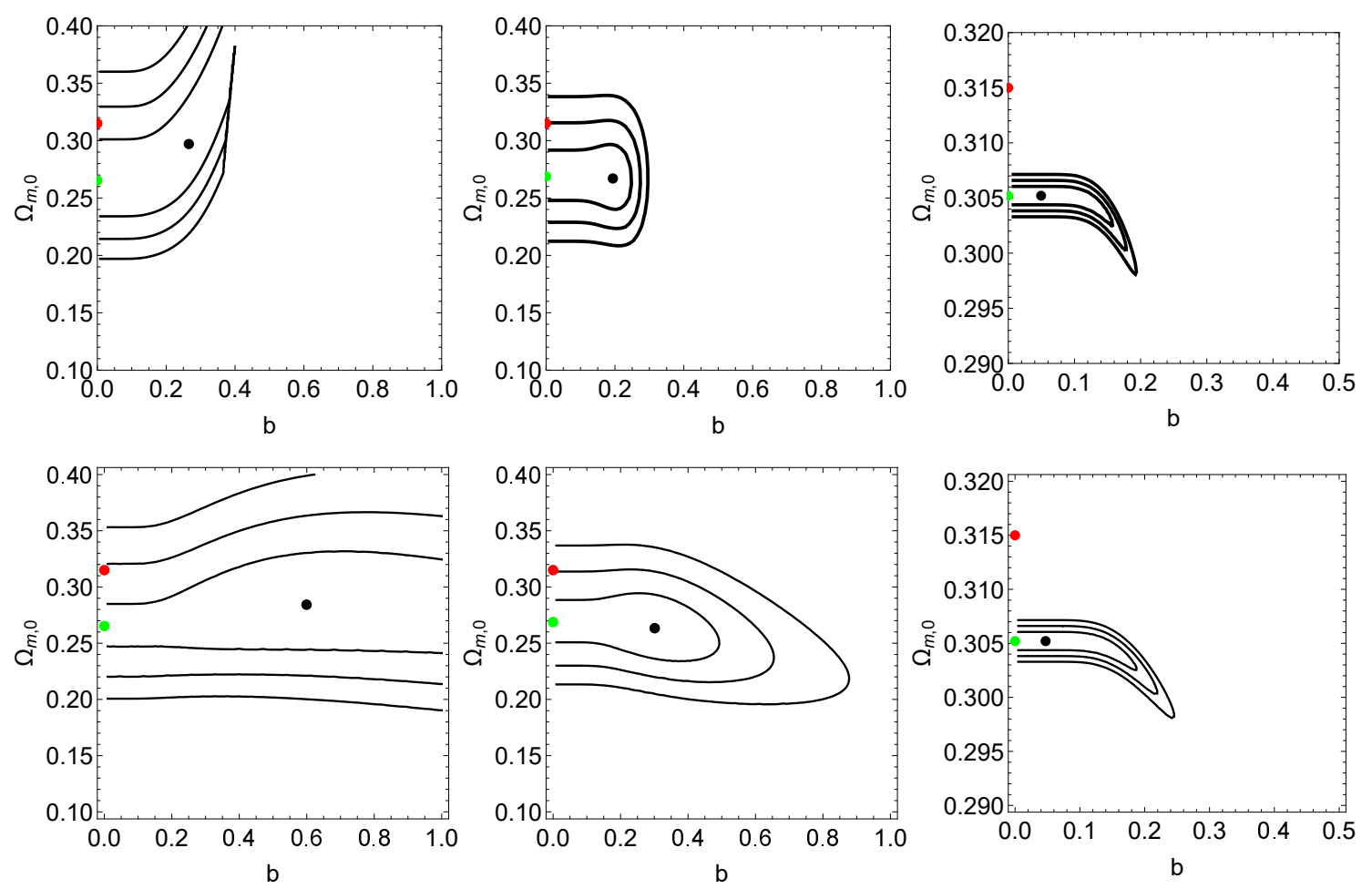

Figure 3. Likelihood contours in the case of $f_{2} \mathrm{CDM}$ (bottom panel) and $f_{3} \mathrm{CDM}$ (top panel) models. From left to right we have (a) $H(z)$ data, (b) $H(z) / \mathrm{QSO} / \mathrm{SNIa}$ and (c) $H(z) / \mathrm{QSO} / \mathrm{SNIa} / \mathrm{CMB}_{\text {shift }}$. The red point corresponds to the Planck $\Lambda$ CDM model [66], while our best fit solutions are given by green $(\Lambda \mathrm{CDM})$ and black $\left(f_{2,3} \mathrm{CDM}\right)$ points respectively.

as well as the ideal Hubble function $H_{\mathrm{r} e}\left(z_{\mathrm{ran}}\right)$ from the reference $f_{1} \mathrm{CDM}$ model. Then we derive the deviation of the observed Hubble parameter from the fiducial $f_{1} \mathrm{CDM}$ model by randomly sampling the distribution of the differences $\delta H=\left|H_{D}-H_{\text {ref }}\right|$.

- In order to produce the mock $H(z)$ data $^{2}$ the corresponding mock Hubble parameter $H_{\mathrm{MC}}$ is chosen from the following normal distribution $\mathcal{N}\left(H_{\mathrm{r} e f}, \sigma_{\mathrm{r} a n}^{2}\right)$, where $\sigma_{\mathrm{r} a n}=\sqrt{\sigma_{H}^{2}+\delta H^{2}}$. Therefore, for each mock entry we provide the following simulated triad $\left\{z_{\mathrm{ran}}, H_{\mathrm{MC}}, \sigma_{\mathrm{ran}}\right\}_{j}$, where $j=1, . . N$ and $N \in[38,120]$.

In Fig. 4 we present the ratio FoM/FoM 38 versus $N$, for which we have performed 100 MonteCarlo realizations for each chosen number $(N=38,40, . .120)$ of the simulated $H(z)$ data. Notice that $\mathrm{FoM}_{38}$ is the Figure-of-Merit of the observed $H(z)$ data. Using standard linear regression we obtain the following relation:

$$
\frac{F o M}{F o M_{38}}=(0.023 \pm 0.0001) N+0.200 \pm 0.018
$$

Based on these new forecasts we argue that it is realistic to expect that a future sample of $\sim 100-$ $120 H(z)$ measurements will increase the present FoM of the $f_{1} \mathrm{CDM}$ model by a factor of $\sim 2.5-$

\footnotetext{
${ }^{2}$ We sample the number of mock data $N \in[38,120]$ in steps of 10 .
} 


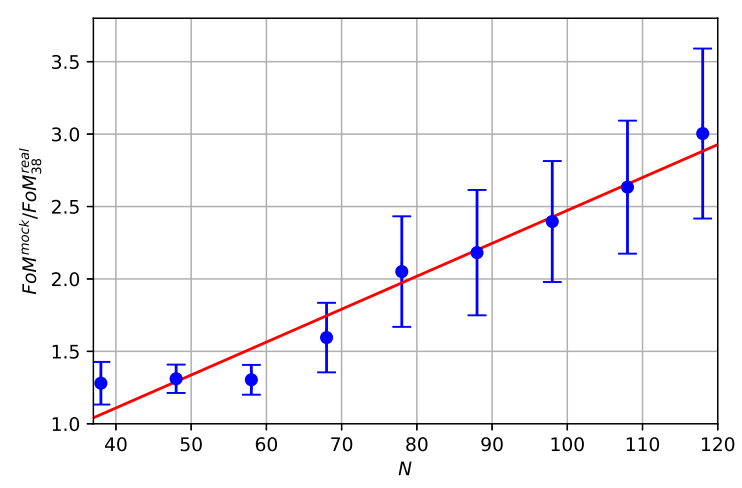

Figure 4. The $F o M / F_{o M}$ as a function of the number of entries in the mock $H(z)$ sample. The quantity $F_{o M_{38}}$ is the Figure-of-Merit of the current $H(z)$ data. The reference model is the power-law $f_{1}$ CDM model (3.5) with $\left(\Omega_{m 0}, b, H_{0}\right)=(0.259,0.121,68.603)$.

3. In order to visualize this improvement in Fig. 5 we present the contours of one simulation of $100 H(z)$ measurements in the $b-\Omega_{m 0}$ plane (red-scale contours). On top on that we show the corresponding contours resulting from the current $H(z)$ sample. The improvement is obvious. Indeed, our Monte Carlo analysis indicates that a future sample of $\sim 100 H(z)$ measurements in the redshift interval $0<z<2.4$, will be an ideal and indispensable tool towards testing the viability of the $f(T)$ gravity models.

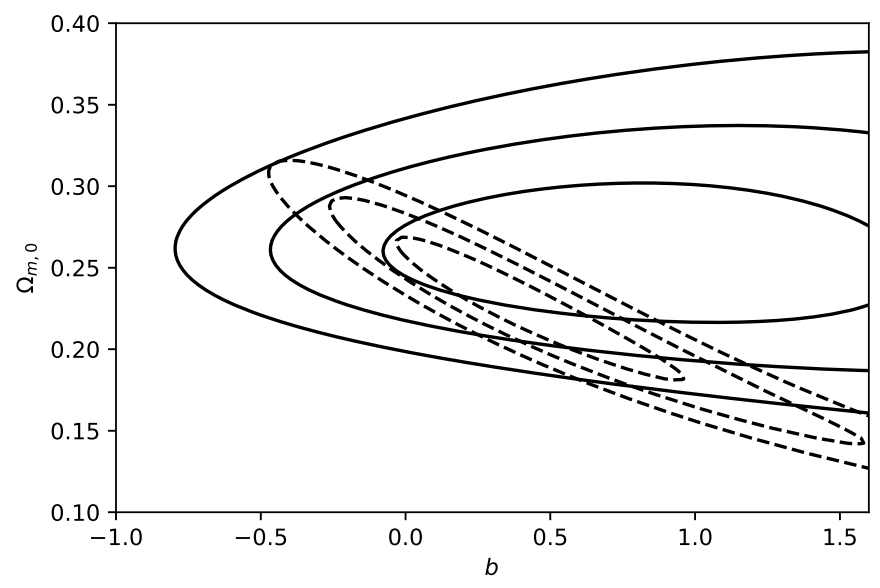

Figure 5. The $1 \sigma, 2 \sigma$ and $3 \sigma$ likelihood contours in the $b-\Omega_{m 0}$ plane resulting from likelihood analysis of the real $H(z)$ data set (black lines) and the mock $H(z)$ sample with $N=100$ entries (red contours). The reference model is the power-law $f_{1} \mathrm{CDM}$ model (3.5) .

\section{Conclusions}

In this work we have extracted observational constraints on the viable $f(T)$ gravity models, using the new $H(z)$ data together with the corresponding covariance matrix and the recently proposed 
statistical method of [68], which is not affected by the value of $H_{0}$ and thus it bypasses the problem of the disagreement in its exact numerical value between Planck and SNIa measurements. In particular, one marginalizes analytically the Hubble constant in the likelihood function using Bayesian statistics, which allows to circumvent the $H_{0}$ value problem and thus to reduce the parameter space without adding numerical complexity. Hence, the aforementioned approach leads to more robust results with respect to previous studies. Finally, apart from the $H(z)$ data, we also proceeded to joint analysis using standard candles such as SNIa and quasi-stellar objects (QSOs), as well as CMB shift parameters data from Planck. To our knowledge, this is the first time that the $H_{0}$-independent method of the $H(z)$ data, the covariance matrix of the $H(z)$ measurements, the JLA and the QSOs data are combined with the Planck CMB shift parameter in constraining the $f(T)$ models.

We considered five $f(T)$ models, each with two independent parameters, with three of these models being viable since they pass the basic observational tests. In all of them we quantified their deviation from $\Lambda \mathrm{CDM}$ cosmology through a sole parameter. Hence, we used the aforementioned observational data in order to fit the model parameters. Furthermore, we applied the AIC criterion in order to compare our results with those of $\Lambda \mathrm{CDM}$ cosmology.

As we saw, the incorporation of more data sets through joint analyses substantially improves the fitting features for all models, and practically all three examined $f(T)$ models, are very efficient and in very good agreement with observations. Among them, $f_{1} \mathrm{CDM}$ model, namely the powerlaw $f(T)$ gravity, is the one with the best fitting behavior, and the one in which a small but non-zero deviation from $\Lambda \mathrm{CDM}$ cosmology is slightly favored (the deviation parameter is non-zero at $1 \sigma$ confidence level). Nevertheless, the corresponding AIC value reveals that $f_{1} \mathrm{CDM}$ model is statistically equivalent with $\Lambda \mathrm{CDM}$ paradigm. On the other hand, for $f_{2} \mathrm{CDM}$ and $f_{3} \mathrm{CDM}$ scenarios, deviation from $\Lambda \mathrm{CDM}$ cosmology is also allowed, however the best-fit values are very close to their $\Lambda \mathrm{CDM}$ one.

Our results are in qualitative agreement with previous observational investigations on $f(T)$ gravity, where the $\mathrm{SNIa} / \mathrm{BAO}$ data alongside the $\mathrm{CMB}$ (shift parameter or power spectrum) data had been used [56-60, 62-65]. However, our analysis improves further the observational constraints with respect to previous studies, since (a) we introduce for the first time the covariance matrix of $H(z)$ data in $f(T)$ models and we use the recently proposed statistical method of [68], which is independent from the Hubble constant problem, and (b) we combine the SNIa data with those of QSOs and thus we manage to trace the Hubble relation in the redshift range $0.07<z<6$. However, the measurements of the distance modulus provided by the QSO data suffer from relatively large errors.

Now, regarding the importance of using direct measurements of the Hubble expansion some considerations are in order at this point. In general, the $H(z)$ data are the only data which are providing a direct measurement of the Hubble expansion as a function of redshift. Obviously, this feature makes them ideal tools for studying the accelerated expansion of the universe.

It is well known that the cosmic acceleration has been traced mainly by SNIa, hence the Hubble relation (distance modulus versus $z$ ) covers the following redshift range $0<z<1.5$ [76, 85]. Also, the binned Pantheon SNIa dataset of [86] lies in the range $0<z<1.6$. Moreover, the geometrical probes utilized to study the cosmic expansion history involve a combination of standard candles (SNIa), standard rulers [clusters, CMB sound horizon detected through Baryon Acoustic 
Oscillations (BAOs; [87, 88]) and the CMB angular power spectrum [66]]. These observational probes trace the integral of the Hubble expansion rate $H(z)$, hence they provide indirect information of the Hubble expansion either up to redshifts of order $z \simeq 1-1.6$ (SNIa, BAO, clusters) or up to the redshift of recombination $(z \sim 1100)$. Clearly, the redshift interval $\sim 1.6-1000$ is not directly probed by the above cosmological data, and as shown in [89] the redshift range $1.6<z<3.5$ plays an important role in cosmic expansion, since different cosmological models give their largest differences in this interval as far as the equation-of-state parameter is concerned. Owing to the fact that the direct $H(z)$ measurements can be computed relatively easily at high redshifts make them, especially those which are located at redshifts $z>1.6$, useful tools in these kind of studies. It is interesting to mention that there are proposed techniques which could expand the direct direct measurements of the Hubble expansion to $z \leq 5$ [90].

To date, a demerit of utilizing alone the present $H(z)$ data-set in constraining the cosmological models, including those of modified gravity, is related with the weak statistical constraints due to small number statistics. However, in order to understand the impact of the current $H(z)$ sample in constraining the models, we have shown that our combined $H(z) / \mathrm{SNIa} / \mathrm{QSO} / \mathrm{CMB}_{\text {shift }}$ statistical analysis (not affected by $H_{0}$ )) correctly reveals the cosmic expansion as provided by the team of Planck [66]. Indeed, in the case of $\Lambda$ CDM model we found $\Omega_{m 0}=0.305 \pm 0.001$ which is in excellent agreement with that of Planck 2016 TT+lowP+lensing data, namely $\Omega_{m 0}=0.308 \pm 0.012$.

Finally, using large sets of Monte Carlo realizations we studied, for the first time, the ability of future measurements of the Hubble expansion to test the viability of the $f(T)$ gravity models. Interestingly, the outcome of the Monte Carlo analysis suggests substantial improvement of the parameter space when we have $\sim 100-120 H(z)$ future measurements. From the observational point of view this is a very realistic prediction in the context of next generation of surveys.

\section{Acknowledgments}

S. Basilakos acknowledges support by the Research Center for Astronomy of the Academy of Athens in the context of the program "Testing general relativity on cosmological scales" (ref. number 200/872). S. Nesseris acknowledges support from the Research Project FPA2015-68048-03-3P [MINECO-FEDER], the Centro de Excelencia Severo Ochoa Program SEV-2016-0597 and from the Ramón y Cajal program through Grant No. RYC-2014-15843. E.N. Saridakis and F. Anagnostopoulos acknowledge support by COST Action (European Cooperation in Science and Technology) "Cosmology and Astrophysics Network for Theoretical Advances and Training Actions".

\section{References}

[1] E. J. Copeland, M. Sami and S. Tsujikawa, Dynamics of dark energy, Int. J. Mod. Phys. (2006) 1753-1936, [hep-th/ 0603057$].$

[2] Y.-F. Cai, E. N. Saridakis, M. R. Setare and J.-Q. Xia, Quintom Cosmology: Theoretical implications and observations, Phys. Rept. 493 (2010) 1-60, [0909.2776].

[3] A. A. Starobinsky, A New Type of Isotropic Cosmological Models Without Singularity, Phys. Lett. B 91 (1980) 99. 
[4] A. H. Guth, The Inflationary Universe: A Possible Solution to the Horizon and Flatness Problems, Phys. Rev. D 23 (1981) 347.

[5] A. D. Linde, A New Inflationary Universe Scenario: A Possible Solution of the Horizon, Flatness, Homogeneity, Isotropy and Primordial Monopole Problems, Phys. Lett. 108B (1982) 389.

[6] V. Sahni and A. Starobinsky, Reconstructing Dark Energy, Int. J. Mod. Phys. 15 (2006) 2105, [astro-ph/0610026].

[7] S. Nojiri and S. D. Odintsov, Introduction to modified gravity and gravitational alternative for dark energy, eConf C 0602061 (2006) 06 Int. J. Geom. Meth. Mod. Phys. 4 (2007) 115 , [hep-th/0601213].

[8] S. Tsujikawa, Modified gravity models of dark energy, Lect. Notes Phys. 800,(2010) 99, [1101.0191]

[9] S. Capozziello and M. De Laurentis, Extended Theories of Gravity, Phys. Rept. 509 (2011) 167-321, [1108.6266].

[10] A. De Felice and S. Tsujikawa, $f(R)$ theories, Living Rev. Rel. 13,(2010), [1002 . 4928].

[11] S. Nojiri and S. D. Odintsov, Unified cosmic history in modified gravity: from $F(R)$ theory to Lorentz non-invariant models, Phys. Rept. 505, 59 (2011), [1011. 054 4].

[12] A. Einstein 1928, Sitz. Preuss. Akad. Wiss. p. 217; ibid p. 224; A. Unzicker and T. Case, Translation of Einstein's attempt of a unified field theory with teleparallelism,[phys i cs / 0503046 ].

[13] K. Hayashi and T. Shirafuji, New general relativity, Phys. Rev. D 19, (1979) 3524.

[14] K. Hayashi and T. Shirafuji, Addendum to "New general relativity", Phys. Rev. D 3312 24, (1982) 3312.

[15] R. Aldrovandi and J. G. Pereira, Teleparallel Gravity, vol. 173. Springer Science+Business Media Dordrecht, 2013, 10.1007/978-94-007-5143-9.

[16] J. W. Maluf, The teleparallel equivalent of general relativity, Annalen Phys. bfseries 525, (2013) 339, [1303.3897].

[17] G. R. Bengochea and R. Ferraro, Dark torsion as the cosmic speed-up, Phys. Rev. D 79 (2009) 124019, [0812 . 1205].

[18] E. V. Linder, Einstein's Other Gravity and the Acceleration of the Universe, Phys. Rev. D 81 (2010) 127301, [1005.3039].

[19] Y.-F. Cai, S. Capozziello, M. De Laurentis and E. N. Saridakis, $f(T)$ teleparallel gravity and cosmology, Rept. Prog. Phys. 79 (2016) 106901, [1511 . 07586].

[20] R. Ferraro and F. Fiorini, Modified teleparallel gravity: Inflation without inflaton, Phys. Rev. D 75 (2007) 084031, [gr-qc/ 0610067 ].

[21] R. Ferraro and F. Fiorini, On Born-Infeld Gravity in Weitzenbock spacetime, Phys. Rev. D 78 (2008) 124019, [0812.1981].

[22] J. B. Dent, S. Dutta, E. N. Saridakis, $f(T)$ gravity mimicking dynamical dark energy. Background and perturbation analysis, JCAP 1101, (2011) 009, [1010 . 2215].

[23] R. Zheng and Q. G. Huang, Growth factor in f(T) gravity, JCAP 1103, (2011) 002, [1010 . 3512].

[24] Y. Zhang, H. Li, Y. Gong and Z. H. Zhu, Notes on $f(T)$ Theories, JCAP 1107, (2011) 015 , [1103.0719]. 
[25] Y. -F. Cai, S. -H. Chen, J. B. Dent, S. Dutta, E. N. Saridakis, Matter Bounce Cosmology with the $f(T)$ Gravity, Class. Quant. Grav. 28, (2011) 215011, [1104.4349].

[26] M. Sharif, S. Rani, F(T) Models within Bianchi Type I Universe, Mod. Phys. Lett. A26, (2011) 1657, [1105.6228].

[27] M. Li, R. X. Miao and Y. G. Miao, Degrees of freedom of $f(T)$ gravity, JHEP 1107, (2011) 108, [1105.5934].

[28] S. Chattopadhyay and U. Debnath, Emergent universe in chameleon, $f(R)$ and $f(T)$ gravity theories, Int. J. Mod. Phys. D 20, (2011) 1135, [ 1105.1091]

[29] S. Capozziello, V. F. Cardone, H. Farajollahi and A. Ravanpak, Cosmography in $f(T)$-gravity, Phys. Rev. D 84, (2011) 043527, [ 1108.2789].

[30] M. H. Daouda, M. E. Rodrigues and M. J. S. Houndjo, Static Anisotropic Solutions in $f(T)$ Theory, Eur. Phys. J. C 72, (2012) 1890, [1109.0528].

[31] C.-Q. Geng, C.-C. Lee, E. N. Saridakis and Y.-P. Wu, Teleparallel dark energy, Phys. Lett. B 704 (2011) 384-387, [1109.1092].

[32] Y. P. Wu and C. Q. Geng, Primordial Fluctuations within Teleparallelism, Phys. Rev. D 86, (2012) 104058, [1110.3099].

[33] H. Wei, X. J. Guo and L. F. Wang, Noether Symmetry in $f(T)$ Theory, Phys. Lett. B 707, (2012) 298, [1112.2270].

[34] W. D. Guo, Q. M. Fu, Y. P. Zhang and Y. X. Liu, Tensor perturbations of $f(T)$-branes, Phys. Rev. D 93, no. 4, (2016) 044002 , [1511.07143]

[35] K. Atazadeh and F. Darabi, $f(T)$ cosmology via Noether symmetry, Eur.Phys.J. C72 (2012) [1112.2824].

[36] Y. C. Ong, K. Izumi, J. M. Nester and P. Chen, Problems with Propagation and Time Evolution in $f(T)$ Gravity, Phys. Rev. D bfseries 88 (2013) 2, 024019, [1303.0993].

[37] J. Amoros, J. de Haro and S. D. Odintsov, Bouncing Loop Quantum Cosmology from $F(T)$ gravity,Phys. Rev. D 87, (2013) 104037 [1305.234 4].

[38] R.-X. Miao, M. Li and Y.-G. Miao, Violation of the first law of black hole thermodynamics in $f(T)$ gravity, JCAP 1111 (2011) 033, [1107.0515].

[39] G. Otalora, Cosmological dynamics of tachyonic teleparallel dark energy, Phys. Rev. D 88, (2013)063505, [1305.5896].

[40] K. Bamba, S. Capozziello, M. De Laurentis, S. ’i. Nojiri and D. Sáez-Gómez, No further gravitational wave modes in $F(T)$ gravity, Phys. Lett. B 727, 194 (2013), [1309.2698].

[41] A. Paliathanasis, S. Basilakos, E. N. Saridakis, S. Capozziello, K. Atazadeh, F. Darabi and M. Tsamparlis, New Schwarzschild-like solutions in $f(T)$ gravity through Noether symmetries, Phys. Rev. D 89, (2014) 104042, [1 402 . 5935]

[42] T. Harko, F. S. N. Lobo, G. Otalora and E. N. Saridakis, $f(T, \mathcal{T})$ gravity and cosmology, JCAP 1412, no. 12, (2014) 021 , [1405.0519]

[43] C. Q. Geng, C. Lai, L. W. Luo and H. H. Tseng, Kaluza-Klein theory for teleparallel gravity, Phys. Lett. B 737, (2014) 248, [1409.1018]

[44] W. El Hanafy and G. G. L. Nashed, The hidden flat like universe, Eur. Phys. J. C 75 (2015) 279, 
[1409.7199].

[45] F. Darabi, M. Mousavi and K. Atazadeh, Geodesic deviation equation in $f(T)$ gravity, Phys. Rev. D 91 (2015) 084023, [1501.00103].

[46] B. Fazlpour, Interacting Quintessence in a New Scalar-Torsion Gravity, Gen. Rel. Grav. 48, no. 12, (2016) 159, [1604.03080].

[47] M. Malekjani, N. Haidari and S. Basilakos, Spherical collapse model and cluster number counts in power law $f(T)$ gravity, Mon. Not. Roy. Astron. Soc. 466, no. 3, (2017) 3488, 1609.01964$].$

[48] G. Farrugia and J. L. Said, Stability of the flat FLRW metric in $f(T)$ gravity, Phys. Rev. D 94, no. 12, (2016) 124054, [1701.00134].

[49] N. Sk, Noether symmetry in $f(T)$ teleparallel gravity, Phys. Lett. B 775, (2017) 100, [1706.00537].

[50] S. Bahamonde, C. G. Böhmer and M. M. Krššák, New classes of modified teleparallel gravity models, Phys. Lett. B 775, (2017) 37 , [1706 . 04920].

[51] M. Hohmann, L. Jarv and U. Ualikhanova, Dynamical systems approach and generic properties of $f(T)$ cosmology, Phys. Rev. D 96, no. 4, ( 2017) 043508, [1706.02376].

[52] A. Conroy and T. Koivisto, The spectrum of symmetric teleparallel gravity, [1710 . 05708].

[53] M. Hohmann, Järv and U. Ualikhanova, Covariant formulation of scalar-torsion gravity, [1801.05786].

[54] Y. F. Cai, C. Li, E. N. Saridakis and L. Xue, $f(T)$ gravity after GW170817 and GRB170817A, Phys. Rev. D 97, no. 10, 103513 (2018), [1801.05827].

[55] L. Iorio and E. N. Saridakis, Solar system constraints on $f(T)$ gravity, Mon. Not. Roy. Astron. Soc. 427 (2012) 1555, [1203. 5781].

[56] P. Wu, H. W. Yu, Observational constraints on $f(T)$ theory, Phys. Lett. B693, 415 (2010), [1006.0674].

[57] G. R. Bengochea, Observational information for $f(T)$ theories and Dark Torsion, Phys. Lett. B695, ( 2011) 405, [1008.3188].

[58] V. F. Cardone, N. Radicella and S. Camera, Accelerating $f(T)$ gravity models constrained by recent cosmological data, Phys. Rev. (2012) 124007, [1204.5294].

[59] S. Nesseris, S. Basilakos, E. N. Saridakis and L. Perivolaropoulos, Viable $f(T)$ models are practically indistinguishable from ACDM, Phys. Rev. D 88 (2013) 103010, [1308 . 6142].

[60] S. Basilakos, Linear growth in power law $f(T)$ gravity, Phys. Rev. D 93, no. 8, (2016) 083007, [1604.00264]

[61] R. C. Nunes, S. Pan and E. N. Saridakis, New observational constraints on $f(T)$ gravity from cosmic chronometers, JCAP 1608 (2016) 011, [1606.04359].

[62] R. C. Nunes, A. Bonilla, S. Pan and E. N. Saridakis, Observational Constraints on $f(T)$ gravity from varying fundamental constants, Eur. Phys. J. C 77 (2017) 230, [1608 . 01960].

[63] S. Capozziello, O. Luongo and E. N. Saridakis, Transition redshift in $f(T)$ cosmology and observational constraints, Phys. Rev. D 91 (2015) 124037, [1503. 02832].

[64] R. C. Nunes, Structure formation in $f(T)$ gravity and a solution for $H_{0}$ tension, [1802 . 02281]

[65] B. Xu, H. Yu and P. Wu, Testing Viable f(T) Models with Current Observations, 
Astrophys. J. 855, no. 2, (2018) 89 .

[66] Planck collaboration, R. Adam et al., Planck 2015 results. I. Overview of products and scientific results, Astron. Astrophys. 594 (2016), [1502 . 01582].

[67] A. G. Riess et al., A 2.4\% Determination of the Local Value of the Hubble Constant, Astrophys. J. 826, no. 1, 56 (2016) , [1604.01424].

[68] F. K. Anagnostopoulos and S. Basilakos, Constraining the dark energy models with H(z) data: An approach independent of $H_{0}$, Phys. Rev. D 97, no. 6, (2018) 063503, [1709.02356].

[69] O. Farooq, F. R. Madiyar, S. Crandall and B. Ratra, Hubble Parameter Measurement Constraints on the Redshift of the Deceleration, acceleration Transition, Dynamical Dark Energy, and Space Curvature, Astrophys. J. 835, no. 1, (2017) 26, [1607.03537] .

[70] Yun Wang and Mi Dai, Exploring uncertainties in dark energy constraints using current observational data with Planck 2015 distance priors, Phys. Rev. D., 94, (2016) 083521, [1607.03537].

[71] Alam, S., et al., The clustering of galaxies in the completed SDSS-III Baryon Oscillation Spectroscopic Survey: cosmological analysis of the DR12 galaxy sample, Mon. Not. R. Soc, , 470, (2017) 2617, [1607.03155 ].

[72] Yu H., Ratra B. and Wang Fa-Yin, Hubble Parameter and Baryon Acoustic Oscillation Measurement Constraints on the Hubble Constant, the Deviation from the Spatially Flat $\Lambda C D M$ Model, the Decelerati on-Acceleration Transition Redshift, and Spatial Curvature, The Astrophysical Journal, (2018), 856.1: 3. [1711.03437]

[73] Weitzenböck R., Invarianten Theorie, Nordhoff, Groningen (1923).

[74] K. Bamba, C.-Q. Geng, C.-C. Lee and L.-W. Luo, Equation of state for dark energy in $f(T)$ gravity, JCAP 1101 (2011) 021, [1011.0508].

[75] P. Wu and H. W. Yu, $f(T)$ models with phantom divide line crossing, Eur. Phys. J. C 71, (2011) 1552, [1008.3669].

[76] M. Betoule et al. [SDSS Collaboration], Improved cosmological constraints from a joint analysis of the SDSS-II and SNLS supernova samples, Astron. Astrophys. 568, A22 (2014), [1401. 4064 ].

[77] G. Risaliti and E. Lusso, A Hubble diagram for quasars, Astroph. J.,815, no. 1 (2015), [1505.07118].

[78] C. Roberts, K. Horne, A. O. Hodson and A. D. Leggat, Tests of $\Lambda$ CDM and Conformal Gravity using GRB and Quasars as Standard Candles out to z 8, [1711.10369].

[79] S. Nesseris \& L. Perivolaropoulos, Comparison of the legacy and gold type Ia supernovae dataset constraints on dark energy models, Phys. Rev. D., 72, 123519, [0 511040 ].

[80] R. Chavez, M. Plionis, S. Basilakos, R. Terlevich, E. Terlevich, J. Melnick, F. Bresolin and A. L. Gonzalez-Moran, Constraining the dark energy equation of state with H II galaxies, Mon. Not. Roy. Astron. Soc. 462, no. 3, (2016) 2431, [1607. 06458$].$

[81] H. Akaike, A new look at the statistical model identification, IEEE Transactions on Automatic Control, 19, (1974) 716.

[82] A. R. Liddle, Information criteria for astrophysical model selection, Mon. Not. Roy. Astron. Soc. 377, (2007) L74, [0701113].

[83] K. P. Burnham, D. R. Anderson, Model selection and multimodel inference: a practical 
information-theoretic approach, 2nd edn. Springer, New York (2002).

[84] K. P. Burnham, D. R. Anderson, Multimodel inference: Understanding AIC and BIC in Model Selection, Sociol. Meth. \& Res., 33, (2004) 261.

[85] N. Suzuki et al., The Hubble Space Telescope Cluster Supernova Survey: V. Improving the Dark Energy Constraints Above z>1 and Building an Early-Type-Hosted Supernova Sample, Astrophys. J. 746 (2012), 85, [1105.3470].

[86] D. M. Scolnic et al., The Complete Light-curve Sample of Spectroscopically Confirmed SNe Ia from Pan-STARRS1 and Cosmological Constraints from the Combined Pantheon Sample, Astrophys. J. 859 (2018) no.2, 101, [1710.00845];

The numerical data of the full Pantheon SnIa sample are available at http://dx.doi.org/10.17909/T95Q4X, https://archive.stsci.edu/prepds/pslcosmo/index.html.

[87] C. Blake et al., The WiggleZ Dark Energy Survey: mapping the distance-redshift relation with baryon acoustic oscillations, Mon. Not. Roy. Astron. Soc. 418, 1707 (2011), [1108.2635].

[88] S. Alam et al. [BOSS Collaboration], The clustering of galaxies in the completed SDSS-III Baryon Oscillation Spectroscopic Survey: cosmological analysis of the DR12 galaxy sample, Mon. Not. Roy. Astron. Soc. 470, no. 3, 2617 (2017), [1607.03155].

[89] M. Plionis, R. Terlevich, S. Basilakos, F. Bresolin, E. Terlevich, J. Melnick and R. Chavez, A Strategy to Measure the Dark Energy Equation of State using the HII galaxy Hubble Relation \& X-ray AGN Clustering: Preliminary Results, Mon. Not. Roy. Astron. Soc. 416, 2981 (2011), [1106.4558].

[90] P. S. Corasaniti, D. Huterer and A. Melchiorri, Exploring the Dark Energy Redshift Desert with the Sandage-Loeb Test, Phys. Rev. D 75, 062001 (2007), [astro-ph/0701433 ]. 